\title{
Critical role of Emx2 in the pluripotency - differentiation transition in male gonocytes via regulation of FGF9/NODAL pathway
}

\author{
Ma Tian-Zhong ${ }^{1}$, Chen $\mathrm{Bi}^{1}$, Zhang Ying ${ }^{2}$, Jing Xia ${ }^{1}$, Peng Cai-Ling ${ }^{1}$, Zhang Yun-Shan ${ }^{1}$, \\ Huang Mei-Wen ${ }^{1}$ and Niu Yan-Ru ${ }^{3}$ \\ ${ }^{1}$ Reproductive Medicine Center, Affiliated Hospital of Guangdong Medical College, Zhanjiang, \\ Guangdong, China, ${ }^{2}$ Department of Gynecology and Obstetrics, Affiliated Hospital of Guangdong Medical College, \\ Zhanjiang, Guangdong, China, and ${ }^{3}$ Laboratory of Minimally Invasive Orthopaedic, Guangdong Medical College, \\ Zhanjiang, Guangdong, China
}

Correspondence should be addressed to Niu Yan-Ru; Email: matzgmc@163.com

\begin{abstract}
Emx2 deletion impairs the growth and maintenance of the genital ridge. However, its role in subsequent germ cell differentiation during embryonic stages is unknown. Using a tamoxifen-inducible Cre-loxP mouse model (Emx $2^{\text {flox/flox }}, C_{\text {re- }} E R^{T M}$, hereafter called as $E m x 2$ knockdown), we showed that germ cell differentiation was impaired in Emx2-knockdown testes. Representative characteristics of male germ cell differentiation, including a reduced ability to form embryonic germ (EG) cell colonies in vitro, down-regulation of pluripotency markers and G1/G0 arrest, did not occur in Emx2-knockdown testes. Furthermore, FGF9 and NODAL signalling occurred at abnormally high levels in Emx2-knockdown testes. Both blocking FGF9 signalling with SU5402 and inhibiting NODAL signalling with SB431542 allowed germ cells from Emx2-knockdown testes to differentiate in vitro. Therefore, EMX2 in somatic cells is required to trigger germ cell differentiation in $\mathrm{XY}$ foetuses, posterior to its previously reported role in the growth and maintenance of the genital ridge.

Reproduction (2016) 151 673-681
\end{abstract}

\section{Introduction}

Primordial germ cells in embryonic gonads respond to molecular signals from the surrounding somatic cells that trigger their differentiation along the spermatogenic or oogenic pathway (Looijenga et al. 2011). Retinoic acid (RA) induces the expression of simulated by retinoic acid 8 (STRA8) to initiate meiosis in female embryonic gonads at approximately E13.5 (Baltus et al. 2006, Bowles et al. 2006, Koubova et al. 2006). In a testicular environment, RA is prevented from inducing meiosis by the action of the degrading p450 enzyme CYP26B1 (Bowles et al. 2006, Koubova et al. 2006). Fibroblast growth factor 9 (FGF9) also suppresses entry into meiosis, maintains expression of pluripotency-related genes and up-regulates markers associated with male germ cell fate, including Nanos2 (Barrios et al. 2010, Bowles et al. 2010). Recent studies have suggested that NODAL signalling works directly on male germ cells to induce their differentiation (Souquet et al. 2012, Spiller et al. 2012, Wu et al. 2013). Souquet and coworkers showed that NODAL signalling reinforces the male fate and prevents meiosis in embryonic germ cells (Souquet et al. 2012). However, Spiller and coworkers showed that NODAL maintains the pluripotency of germ cells and delays differentiation, with no effect on meiosis (Spiller et al. 2012). Wu et al. further suggested that NODAL signalling acts downstream of the somatic cell signal FGF9 to promote male germ cell fate and suppress meiosis (Wu et al. 2013).

The balance between pluripotency regulation and cell fate commitment is crucial. Defects in pluripotency result in insufficient spermatogonia for fertility, whereas escape from commitment causes testicular germ cell tumours (Looijenga et al. 2011). Germ cell differentiation is associated with down-regulation of pluripotency regulators (POU5F1 (OCT4), SOX2, NANOG and DPPA4), appearance of differentiation indexes (including up-regulation of the male fate marker gene Nanos2 (Tsuda et al. 2003), entry into cell cycle arrest (Western et al. 2008) and de novo DNA methylation (Kaneda et al. 2004, Sasaki \& Matsui 2008, Western et al. 2010)). In vivo, both transcriptional repression and chromatin remodelling block germ cells from acquiring a fully pluripotent stem cell state (Durcova-Hills \& Surani 2008). Although the developmental potency of foetal germ cells is restricted to the germ lineage, foetal germ cells at a bipotential stage (before E11.5) can dedifferentiate into 
embryonic germ (EG) cells that possess pluripotency similar to embryonic stem (ES) cells in vitro (Labosky et al. 1994, Kimura et al. 2005, 2008) when exposed to exogenous signalling molecules, leukaemia inhibitory factor (LIF), stem cell factor (SCF) and basic fibroblast growth factor (bFGF); however, this ability is lost after differentiation (Matsui et al. 1992, Resnick et al. 1992, Durcova-Hills \& Surani 2008, Matsui \& Tokitake 2009).

The homeobox gene Emx2 is a mouse homologue of the Drosophila head gap gene empty spiracles (ems). EMX2 is expressed in the epithelial components of the developing urogenital system and is required for growth and maintenance of the genital ridge (Miyamoto et al. 1997). Emx2 deletion impairs the migration of epithelial cells through the basement membrane to form the genital ridge (Kusaka et al. 2010). However, it is unknown whether Emx2 regulates subsequent germ cell differentiation during the embryonic stage. In this study, we addressed this question by employing a tamoxifeninducible Cre-loxP mouse model ( $E m \times 2^{\text {floxflox }}, C r e-E r^{T M}$, hereafter called as Emx2 knockdown).

Various aspects of male germ cell differentiation were disrupted in Emx2-knockdown testes. First, the germ cells in Emx2-knockdown testes showed a high propensity to form pluripotent EG cell lines in vitro at E13.5, which is no longer possible for wild-type differentiated germ cells at the same stage. Secondly, the expression of the pluripotent regulators (POU5F1, SOX2, NANOG and DPPA4) was still detected after E15.5. Thirdly, G1/G0 arrest was not observed in Emx2-knockdown testes. We used in vitro culture models together with inhibitors to demonstrate that these effects were mediated at least in part by abnormally high levels of FGF9 and NODAL signalling. Our findings expand the known developmental roles of EMX2 by demonstrating its role in the pluripotency-differentiation transition and its interaction with the FGF9/NODAL pathway.

\section{Materials and methods}

\section{Mouse breeding}

All animal research was approved by the committee on animal care at the Guangdong Medical College (Permit number: SYXK (Guangdong): 2008-0007). All mice were maintained on a C57BL/6;129/SvEv-mixed background. A targeting vector containing Emx2 exon 2 flanked by a loxP site and a loxPNeo cassette was constructed and introduced into mouse embryonic stem (ES) cells (AB1, 129/SvEv) by electroporation (Supplementary Fig. S1A, see section on supplementary data given at the end of this article). Next, ES cell clones containing the targeted Emx2 construct were injected into C57BL/6 (B6) blastocysts to generate chimaeras. The resulting male chimaeras were identified by coat colour and mated with wildtype females to obtain germ line transmission. Tail biopsies of agouti-pigmented F1 animals were genotyped using a primer set specific to the Neo cassette. Emx2 $2^{\text {floxfllox }}$ mice were mated with mice carrying the Cre-Er ${ }^{\mathrm{TM}}$ (Hayashi \& McMahon 2002)

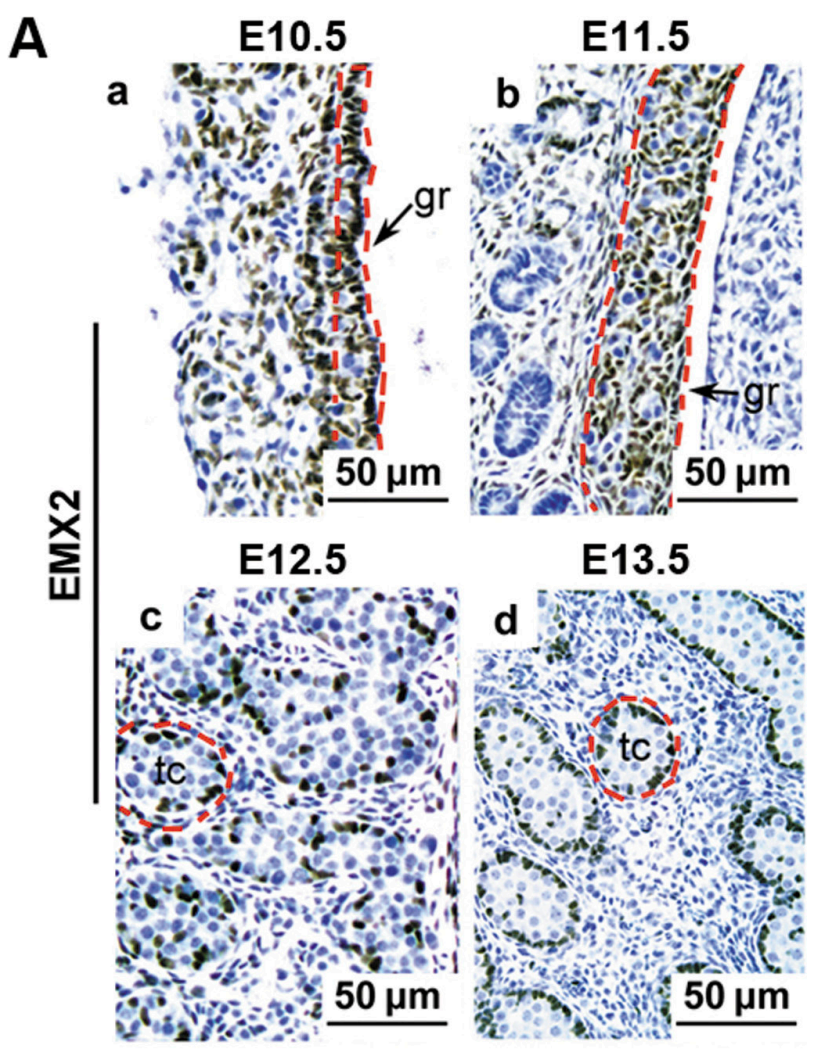

B
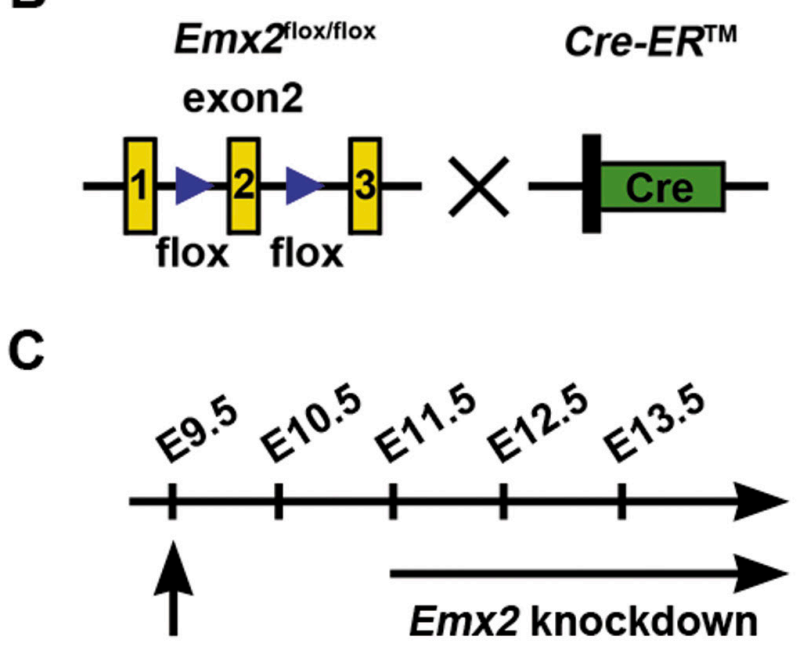

Tamoxifen injection

Figure 1 The tamoxifen-inducible Emx2 knockdown used in this study. (A) Immunohistochemical staining was performed on paraffin sections. Haematoxylin was used for counterstaining. EMX2 was specifically expressed in the somatic cells of the genital ridge at E10.5 and E11.5 ( $a$ and b). EMX2 was localized to Sertoli cells from E12.5 and E13.5 (C and d). gr, genital ridge; tc, testis cord. Scale bar, $50 \mu \mathrm{m}$. (B) Schematic for generating the animals carrying the $E m \times 2^{\text {flox }}$ and the recombined allele in which exon 2 is flanked by loxP sites. (C) To induce Cre recombinase activity, pregnant mice were injected with $12 \mathrm{mg} / 40 \mathrm{~g}$ (body weight) tamoxifen at E9.5, and foetal testes were collected from embryos after E11.5. 
transgene to produce $E m \times 2^{\text {floxflox }}, C r e-E r^{T M}$ offspring. DNA isolated from tail and embryo biopsies was used for genotyping (Fig. S1B). The presence of the floxed allele and Cre transgene was determined by PCR amplification. Mice were genotyped by PCR analysis using primers (5'-GAGCCTGAGGACTGCCA-3' and 5'-GGCTCGCTTGTTCAGTCT-3') to identify the loxP locus and primers (5'-GCCAGGCCACACTACATC- $3^{\prime}$ and 5'-GCGGAGAAAAGCTAGC-3') to identify the exon 2-deleted locus for Emx2 targeting. Tamoxifen (Sigma-Aldrich) was dissolved in corn oil to a final concentration of $20 \mathrm{mg} / \mathrm{mL}$. For detailed characterization of the Cre activity, 3, 6, 9, 12, 13 or $15 \mathrm{mg}$ tamoxifen/40 g of body weight was injected intraperitoneally into pregnant mothers at E9.5. A higher concentration of tamoxifen (>12 mg tamoxifen/4 g body weight)

A

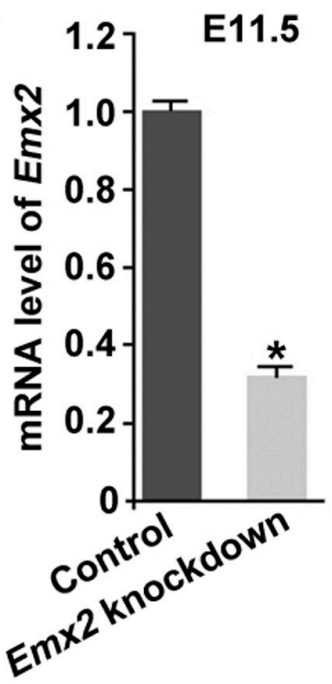

B

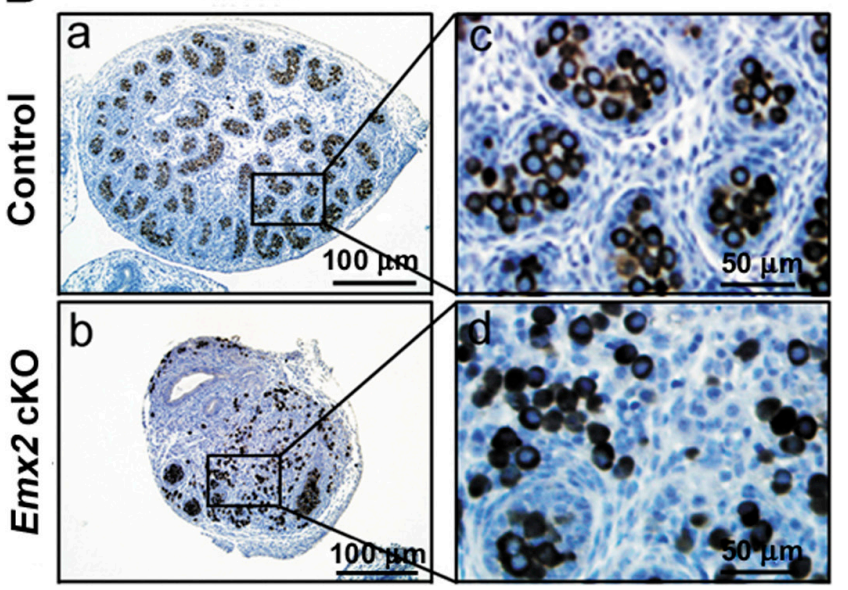

Figure 2 Morphological analysis of $E m \times 2^{\text {floxflox }}, C r e-E r^{T M}$ gonads. (A) The efficiency of tamoxifen-induced Cre recombination was examined by qRT-PCR at E11.5. Compared with wild-type testes, $E m \times 2$ transcription levels were reduced by approximately $70 \%$ in $E m \times 2^{\text {floxflox }}, C r e-E r^{T M}$ testes. ${ }^{*} P<0.05$; Student's $t$-test. (B)

Morphological analysis of $E m \times 2^{\text {flox/flox }}, C r e-E r^{T M}$ gonads at E15.5. DDX4-expressing germ cells were present throughout the gonad, and the basement membrane did not outline testis cords. Scale bar in a and b, $100 \mu \mathrm{m}$. Scale bar in c and d, $50 \mu \mathrm{m}$. caused embryonic lethality. Accordingly, we chose $12 \mathrm{mg}$ tamoxifen/40 g body weight to induce excision of the floxed Emx2 allele (Hayashi \& McMahon 2002). Tamoxifen-treated $E m \times 2^{+/ f l o x}, C r e-E r^{T M}$ mice served as controls in all experiments.

\section{Germ cell-EG dedifferentiation}

Embryonic germ cell culture was performed as described previously with minor modifications (Durcova-Hills \& Surani 2008). Gonads containing germ cells from E11.5 and E13.5 embryos were dissociated into single cells by incubation in $0.05 \%$ trypsin/0.02\% EDTA (Invitrogen) for $5 \mathrm{~min}$ in a $37^{\circ} \mathrm{C}$ water bath, and a cell suspension was obtained by gently pipetting tissues. Germ cells labelled with a C-terminal DDX4/ MVH antibody (Abcam, ab13840; 1:100) were sorted by fluorescence-activated cell sorting (FACS) using an Influx Cell Sorter (BD Biosciences, San Jose, CA, USA). Cells were plated

\section{A}
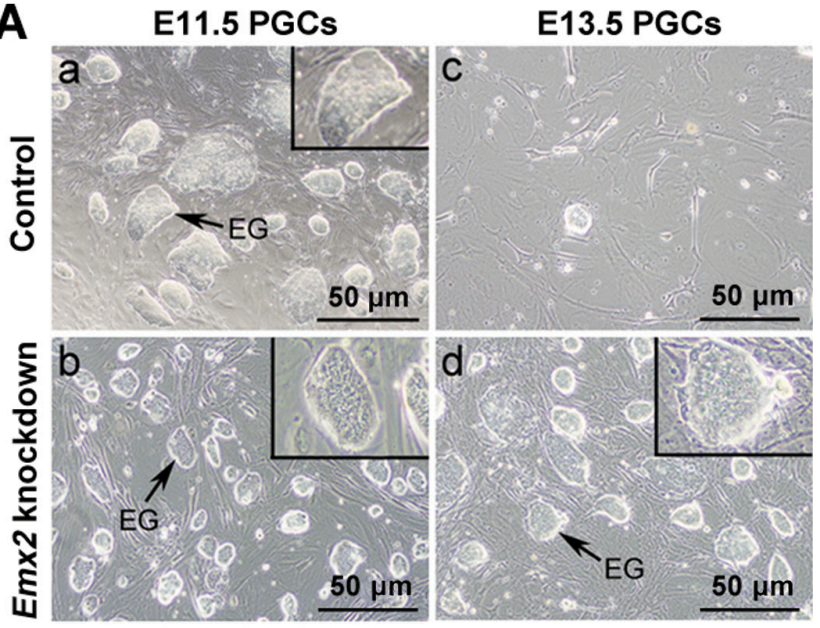

B

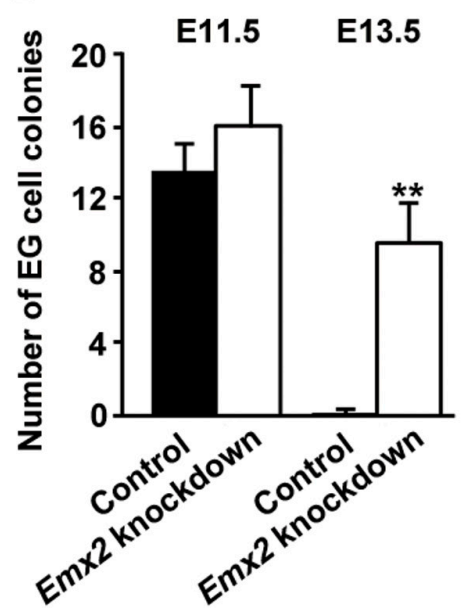

Figure 3 EMX2 determines germ cell pluripotency to form EG clones in vitro. (A) Gonads containing PGCs from E11.5 (a and b) and E13.5 (c and d) control (a and c) or Emx2 knockdown (b and d) testes were isolated and cultured on feeder cells in the presence of essential survival and growth factors for 10 days. Arrows denote EG clones. Scale bar, $50 \mu \mathrm{m}$. (B) Number of EG cell colonies was shown from E11.5 and E13.5 control or Emx2-knockdown testes. ${ }^{* *} P<0.01$; Student's $t$-test. 

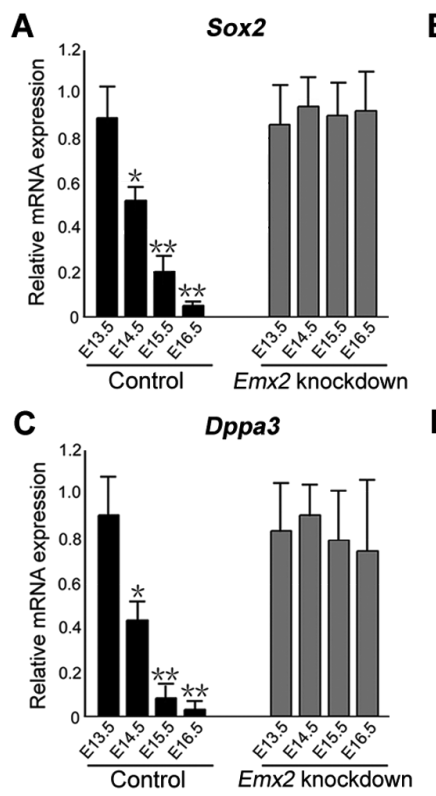

B

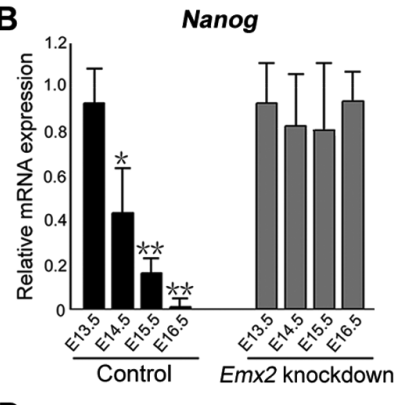

D

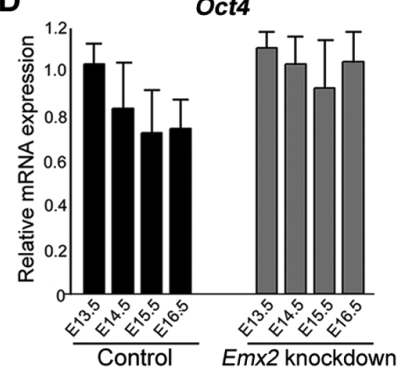

Figure 4 Germ cells in $E m x 2^{\text {floxflox }}, \mathrm{Cre}^{-E r^{T M}}$ testes exhibited prolonged pluripotency. Quantitative analysis of Pou5f1 (A), Nanog (B), Sox2 (C) and Dppa3 (D) mRNA levels in wild-type and Emx $2^{\text {floxflox }}$, Cre-Er ${ }^{T M}$ testes from E13.5 to E16.5. Expression was normalized to $D d x 4$. Three independent biological replicates were induced for each time point. A post hoc multiple comparisons test following ANOVA was used. ${ }^{*} P<0.05,{ }^{* *} P<0.01$.

onto mitotically inactivated (treated with $5 \mu \mathrm{g} / \mathrm{mL}$ mitomycin C (Sigma-Aldrich) for $2 \mathrm{~h}$ the day before use) SI/SI4-m220 feeder cells in 12-well plates with PGC growth media (DurcovaHills \& Surani 2008). Media were changed daily for 10 days until small colonies were observed. EG cells were identified by the characteristic colony morphology of densely packed cells and by staining for pluripotency markers such as POU5F1 (sc-8629, 1:200; Santa Cruz), NANOG (ab80892, 1:200; Abcam), SOX2 (2683-1, 1:100; Epitomics, Burlingame, CA, USA) and c-MYC (ab32072, 1:200; Abcam).

\section{Collection of foetal gonads and organ culture}

Foetal gonads were isolated and cultured as described previously (Livera et al. 2006). The gonads of tamoxifentreated $E m \times 2^{\text {flox fllox }}, \mathrm{Cre}-E r^{T M}$ and $E m \times 2^{+ \text {fflox }}, \mathrm{Cre}-E r^{T M}$ (control) embryos were harvested at E11.5, and feotal sex was determined using PCR amplification of Sry (Koopman et al. 1991). One gonad per embryo was used as the control and was cultured with the appropriate vehicle. The contralateral gonad was cultured with $20 \mu \mathrm{M}$ FGFR-specific tyrosine kinase inhibitor SU5402 (Calbiochem) (Wu et al. 2013) or $20 \mu \mathrm{M}$ specific type I ALK 4/5/7 receptor inhibitor SB431542 (Sigma-Aldrich) (Souquet et al. 2012) for $72 \mathrm{~h}$ before quantitative RT-PCR.

\section{Immunofluorescence and immunohistochemistry}

Embryonic gonads were collected from E12.5 to E18.5. Gonads were dissected in PBS and fixed in 4\% PFA for up to $24 \mathrm{~h}$, stored in $70 \%$ ethanol and embedded in paraffin. Tissue sections ( $5 \mu \mathrm{m})$

were cut and mounted on glass slides. Immunofluorescence and immunohistochemistry of deparaffinized sections were performed as described previously (Chen et al. 2013). Primary antibodies included anti-EMX2 (ab110112, 1:200; Abcam), anti-DDX4 antibody (ab13840, 1:200; Abcam), anti-KI67 (ab15580, 1:400; Abcam), anti-STELLA (sc-67249; Santa Cruz), anti-SOX9 (ab3697, 1:400; Abcam), anti-STRA8 (ab49602; Abcam) and anti-DAZL (MCA2336, 1:200; AbD Serotec (San Diego, CA, USA)). FITC or TRITC-conjugated secondary antibodies were from Jackson ImmunoResearch. The sections were counterstained with DAPI (Sigma-Aldrich) to label the nuclei. To determine the percentage of gonocytes that were positive for KI67, we counted at least ten sections for each group.

\section{Western blot}

Proteins from isolated foetal testes were electrophoresed under reducing conditions in $12 \%$ SDS-PAGE gels and transferred onto nitrocellulose membranes. The blots were blocked in $5 \%$ BSA, incubated overnight at $4{ }^{\circ} \mathrm{C}$ with primary antibody (anti-EMX2; Abcam, ab110112, 1:1000) and incubated with the IRDye 680 or IRDye 800 (LI-COR Bioscience, Lincoln, $\mathrm{NE}$, USA) secondary antibody for $1 \mathrm{~h}$ at room temperature. The specific signals and the corresponding band intensities were evaluated using an Odyssey Infrared Imaging system and software (LI-COR Bioscience, Lincoln, NE, USA).

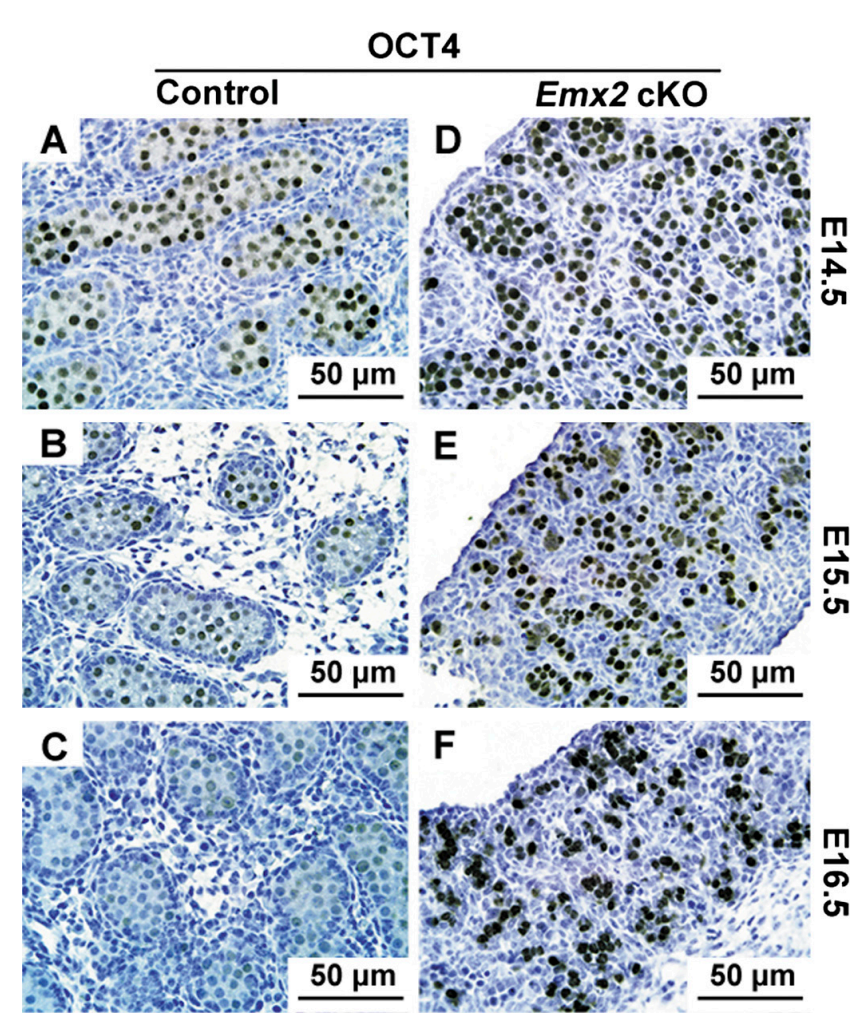

Figure $5 \mathrm{Immunohistochemical} \mathrm{staining} \mathrm{for} \mathrm{POU5F1} \mathrm{protein} \mathrm{in}$ sections of wild-type (A, B and C) and $E m \times 2^{\text {flox/flox }}, C r e-E r^{T M}$ testes (D, E and F). Scale bar, $50 \mu \mathrm{m}$. 


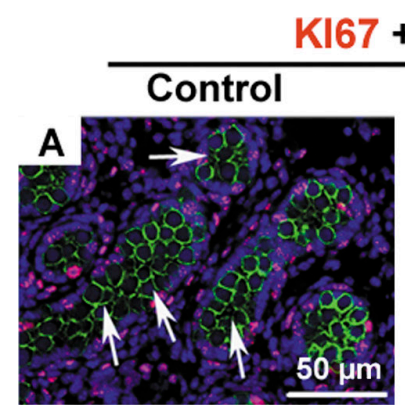

\section{Emx2 knockdown}
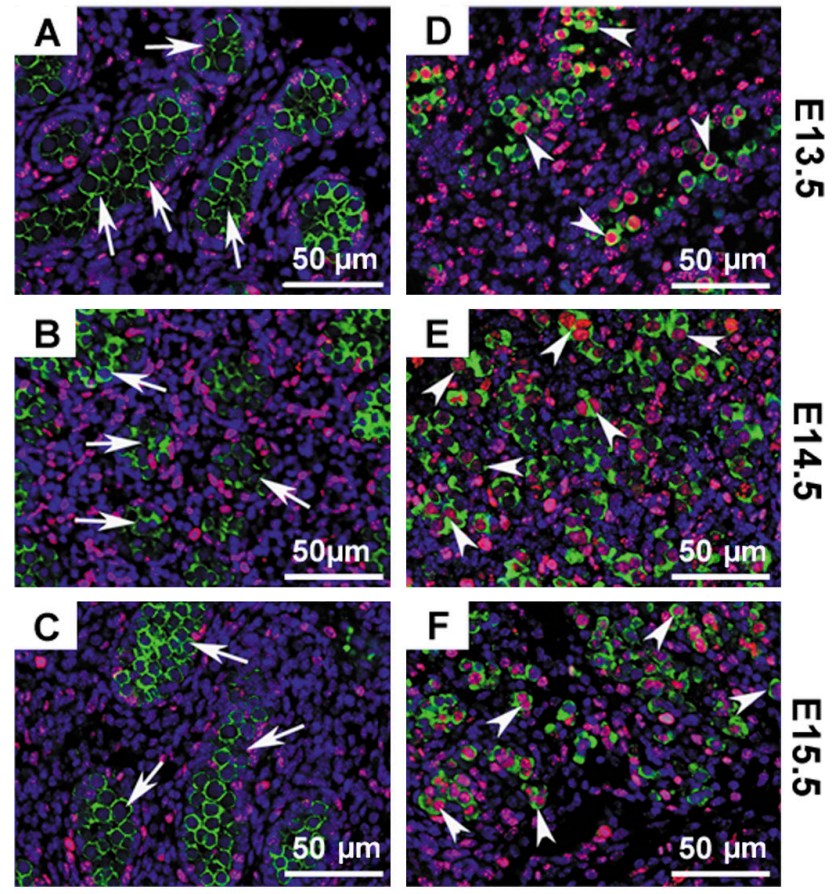

G

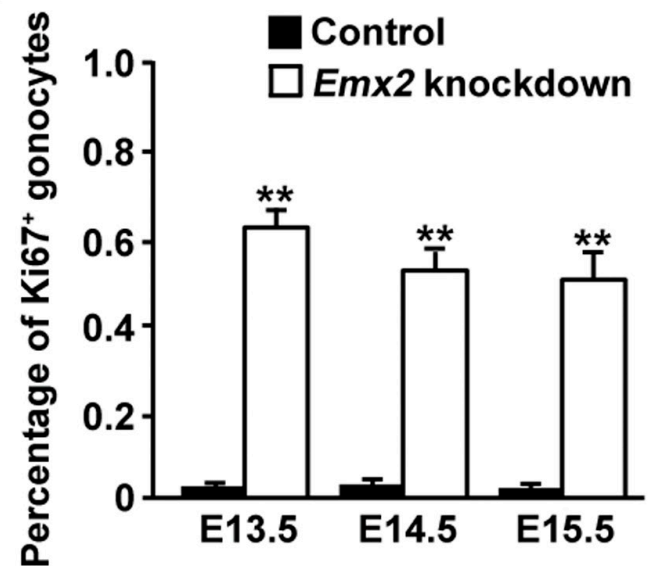

Figure 6 Germ cells in Emx2-knockdown testes did not undergo G1/G0 cell cycle arrest. Germ cells were labelled with DAZL (green) and $\mathrm{KI} 67$ (red) labelled proliferating cells. The KI67 signal was absent in most of the germ cells of control testes after E13.5 (A, B and C, arrows). Germ cells in Emx2-knockdown testes were still KI67 positive from E13.5 to E15.5 (D, E and F, arrowheads). Nuclei were stained with DAPI (blue). Scale bar, $50 \mu \mathrm{m}$. (G) Percentage of KI67-positive gonocytes in control and Emx2-knockdown testes at E13.5, E14.5 and E15.5. ${ }^{* * P}<0.01$; Student's $t$-test.

\section{Quantitative (q)RT-PCR}

Total RNA was extracted from foetal testes using a Qiagen RNeasy kit in accordance with the manufacturer's instructions. To quantify gene expression, real-time SUBR Green assays were performed with the isolated RNA (Tian Gen, Beijing, China). The results were analysed by the $2^{-\Delta c t}$ method. Gene expression was quantified relative to Gapdh or $D d \times 4(M v h)$ expression. Primer sequences are listed in Table S1.

\section{Statistical analysis}

The results of three experiments are presented as the mean \pm S.E.M. The data were evaluated for significant differences using Student's $t$-test. When comparing multiple samples, a post hoc multiple comparisons test following ANOVA was used. A $P$-value $<0.05$ was considered to be significant, and a $P$-value $<0.01$ was considered to be highly significant. Bar graphs were plotted in MS Excel.

\section{Results}

\section{Tamoxifen-inducible Emx2 knockdown after E11.5}

EMX2 was predominantly expressed in the somatic cells of the genital ridge at E10.5 and E11.5 (Fig. 1A; $a$ and b). Afterwards, EMX2 was specifically localized
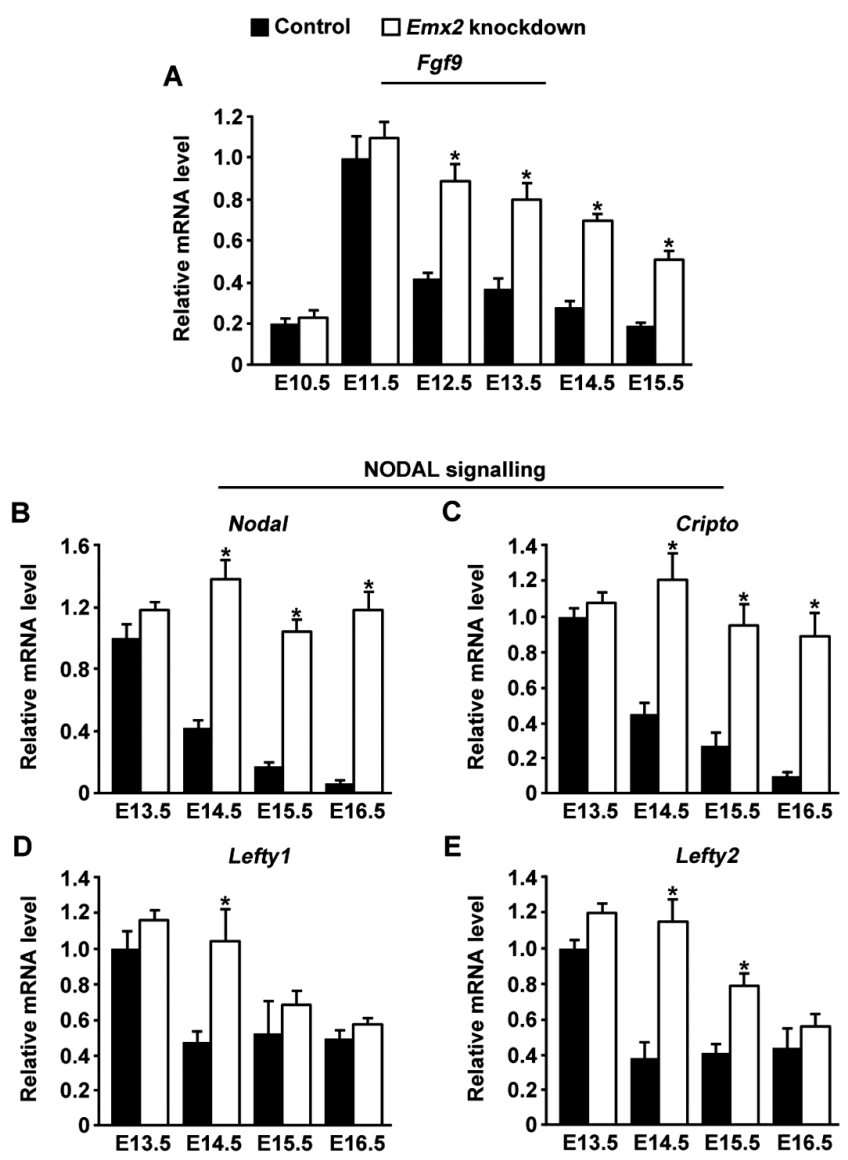

Figure 7 FGF9 and NODAL signalling was hyperactivated in Emx2-knockdown testes. (A) qRT-PCR analysis of Fgf9 from E10.5 to E15.5 between control and Emx2-knockdown testes. Gapdh was used as normalization control. (B, C, D and E) QRT-PCR analysis of Nodal (B), Cripto (C), Lefty1 (D) and Lefty2 (E) from E13.5 to E16.5 control and $E m \times 2$-knockdown testes. $D d \times 4$ was used as normalization control. Three independent biological replicates were induced for each time point. ${ }^{*} P<0.05$; Student's $t$-test. 
Control XY $\square$ Emx2 knockdown $X Y$

Control XY+SU5402
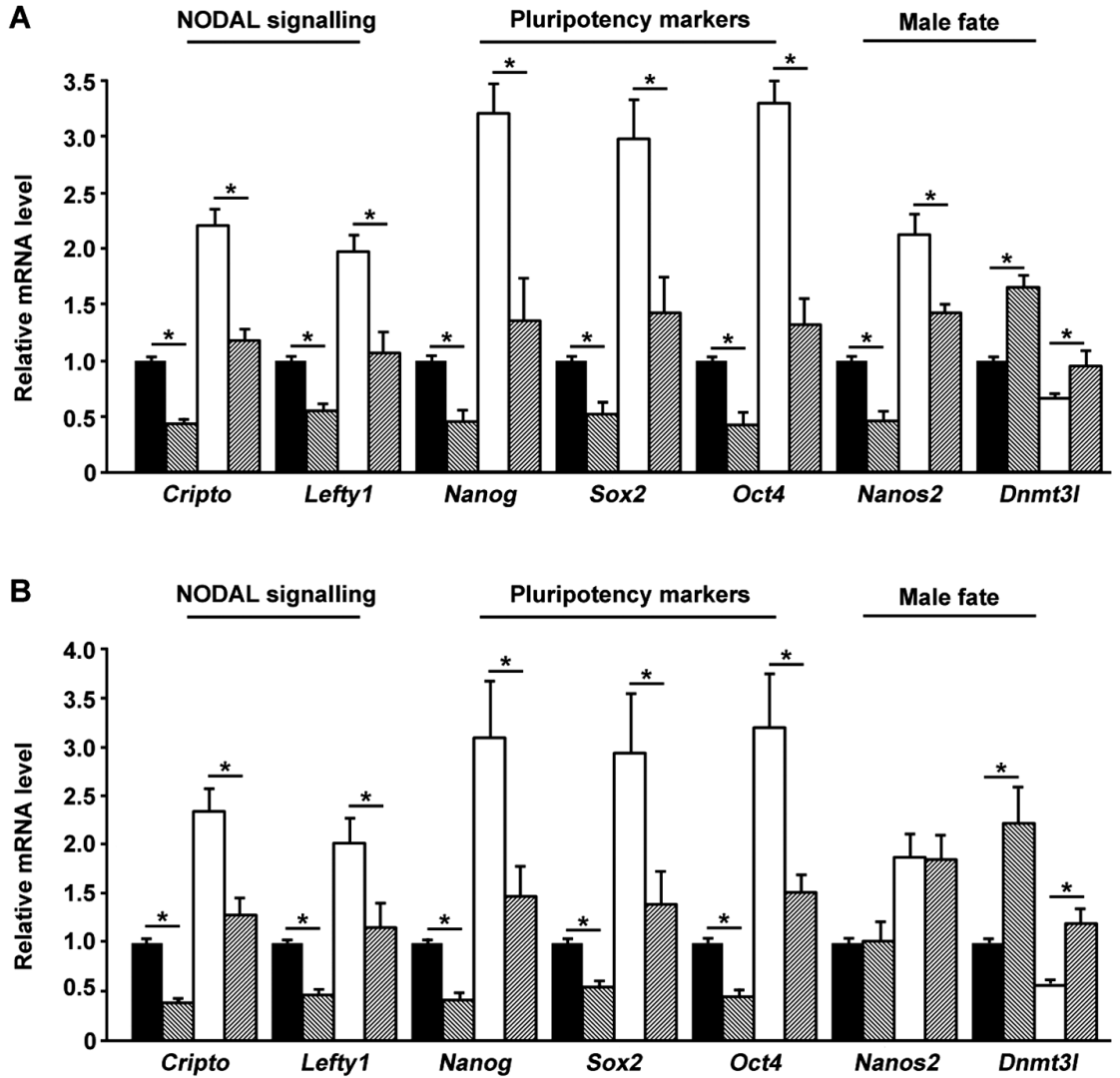

Figure 8 Blocking FGF9 or NODAL signalling allowed germ cells in Emx2-knockdown testes to differentiate. E11.5 Emx2-knockdown and control testes were treated with SU5402 $(20 \mu \mathrm{M})(\mathrm{A})$ or SB431542 $(20 \mu \mathrm{M})(\mathrm{B})$, and Cripto, Lefty1, Nanos2, pluripotency-related genes Nanog, Sox2 and Pou5f1 as well as the differentiation-related gene Dnmt3/ were analysed by qRT-PCR after $72 \mathrm{~h}$ of culture. $D d \times 4$ was used as normalization control. Three independent biological replicates were induced for each time point. ${ }^{*} P<0.05$; Student's $t$-test. to Sertoli cell nuclei at E12.5 and E13.5 (Fig. 1A; $\mathrm{c}$ and $\mathrm{d}$ ). In addition, we detected a band of the predicted EMX2 size in testes from E11.5 to E15.5 by western blot (Fig. S2A). We performed double staining for EMX2 and SOX9 (a somatic cell marker) and EMX2 and STELLA (a germ cell maker) at E11.5. As expected, EMX2 was specifically expressed in somatic cells but not in gonocytes (Fig. S2B). To investigate the role of EMX2 during embryonic gonad development, we utilized a tamoxifen-inducible Cre-loxP mouse model (Emx $\left.2^{\text {flox/flox }}, \mathrm{Cre}-E r^{T M}\right)$ (Fig. 1B). To induce Cre recombinase activity, pregnant mice were injected with $12 \mathrm{mg} / 40 \mathrm{~g}$ (body weight) tamoxifen at E9.5, and foetal testes were collected from the embryos after E11.5 (Fig. 1C). The Emx2 mRNA level was reduced by approximately $70 \%$ in Emx2-knockdown testes relative to controls at E11.5 (Fig. 2A). EMX2 was successfully deleted in Emx2-knockdown testes, as demonstrated by immunohistochemistry (Fig. S2C). Morphological analysis revealed that the basement membrane poorly outlined the cords, and DDX4expressing germ cells were present throughout the testes in the Emx2-knockdown mice at E15.5 (Fig. 2B). Emx2-knockdown mice exhibited perinatal lethality.

\section{Germ cells in Emx2-knockdown testes maintain pluripotency instead of differentiation}

The ability of PGCs to generate EG cell colonies is restricted to the undifferentiated PGCs before E11.5 (Matsui \& Tokitake 2009). To examine the differentiation status, XY gonads containing germ cells from E11.5 and E13.5 control or Emx2-knockdown embryos were isolated and cultured under EG colony-forming conditions. EG cells were identified by the characteristic colony morphology of densely packed cells and by staining for the pluripotency markers POU5F1, NANOG, SOX2 and C-MYC (Fig. S3). No significant difference in the formation of primary EG cell colonies was observed between control testes and Emx2-knockdown testes at E11.5 (Fig. 3A; a and b). After E11.5, PGCs differentiated along the spermatogenic or oogenic pathway. As expected, the efficiency of deriving EG cells from E13.5 germ cells in control testes was vanishingly low (Fig. 3A; c). Significant reduction in EG cell colony formation was observed in E13.5 Emx2-knockdown testes relative to control testes (Fig. 3A; d, 3B). Collectively, germ cells maintain pluripotency and the ability to dedifferentiate into EG cells in Emx2-knockdown testes. 
Next, we examined the expression of pluripotency markers in Emx2-knockdown testes from E13.5 to E16.5 by qRT-PCR (Fig. 4). Compared with control testes, germ cells in Emx2-knockdown testes continued to express Sox2, Nanog and Dppa3 at high levels from E13.5 to E16.5 (Fig. 4A, B and C). Although the Pou5f1 mRNA level remained unchanged (Fig. 4D), POU5F1 protein levels were significantly reduced after E14.5 in wild-type testes, as demonstrated by staining (Fig. 5A, B and C). We observed that germ cells extended the period of POU5F1 protein production in Emx2-knockdown testes (Fig. 5D, E and F).

In wild-type testes, most germ cells were negative for the active cell cycle marker KI67 after E13.5 (Fig. 6A, B and C), indicating that they had entered mitotic arrest. By contrast, the percentage of KI67-positive male gonocytes was significantly increased in Emx2-knockdown testes from E13.5 to E15.5 (Fig. 6D, E, F and G).

\section{Hyperactivation of FGF9-NODAL signalling in Emx2- knockdown testes}

Using qRT-PCR, we observed that the Fgf9 mRNA level was significantly increased in Em×2-knockdown testes compared with control testes from E12.5 to E15.5 (Fig. 7A). FGF9 stimulates Nanos2 expression in ex vivo gonad cultures (Barrios et al. 2010, Bowles et al. 2010). As expected, the Nanos 2 mRNA level was significantly up-regulated in Emx2-knockdown testes, relative to control testes from E12.5 to E16.5 (Fig. S4A). Furthermore, the mRNA levels of Nodal and the NODAL downstream target genes Cripto, Lefty 1 and Lefty 2 were substantially down-regulated after E13.5 in control testes. However, Nodal, Cripto, Lefty 1 and Lefty 2 were significantly overexpressed in Emx2-knockdown testes, relative to control testes between E14.5 and E16.5 (Fig. 7B, C, D and E).

\section{Blocking either FGF9 or NODAL signalling allows germ cells to differentiate in Emx2-knockdown testes}

To determine the effects of high levels of FGF9 and NODAL signalling on the arrest of differentiation phenotype, the FGFR tyrosine kinase inhibitor SU5402 (Mohammadi et al. 1997) and the ALK4/5/7 inhibitor SB431542 (Inman et al. 2002) were added to E11.5 control and Emx2-knockdown testes respectively (Fig. 8). The expression of NODAL target genes such as Cripto and Lefty 1 was significantly decreased in the SU5402 or SB431542 treatment conditions. The mRNA levels of Nanos2 and pluripotency-related genes such as Nanog, Sox2 and Pou5f1 were significantly reduced in SU5402treated control and Emx2-knockdown testes after $72 \mathrm{~h}$ of ex vivo culture, accompanied by increased expression of the differentiation-related gene Dnmt3l, compared with the untreated groups (Fig. 8A). We also observed the reduced expression of pluripotency-related genes such as Nanog, Sox2 and Pou5f1, and, conversely, the increased expression of the differentiation-related gene Dnmt3/ in control and Emx2-knockdown testes after SB431542 treatment (Fig. 8B).

\section{Discussion}

EMX2 is required for embryonic gonad development, as $E m \times 2$ deletion leads to urogenital development defects (Miyamoto et al. 1997, Kusaka et al. 2010). In this study, we used a conditional knockdown strategy $\left(E m \times 2^{\text {flox fllox }}\right.$, $\left.\mathrm{Cre}-E r^{T M}\right)$ to knock down $E m \times 2$ at approximately E11.5 (after genital ridge formation) and then examined the consequences of this deletion on male germ cell differentiation. We showed that various aspects of male germ cell differentiation were blocked in these knockdown mice. In tamoxifen-treated $E m \times 2^{\text {floxflox }}$, $\mathrm{Cre}-\mathrm{Er}$ foetal testes, germ cells expressed pluripotency genes, including Sox2, Nanog, and Pou5f1, at high levels after E14.5, when their expression is normally down-regulated to allow spermatogenic differentiation to proceed. As a result, germ cells in Emx2-knockdown foetal testes were still proliferating at E13.5, whereas germ cells in control testes had become mitotically quiescent at this stage. Consistently, germ cells in Emx2-knockdown testes retained the ability to form EG colonies in vitro at E13.5, when control germ cells have already lost this ability.

Interestingly, although germ cells in Emx2-knockdown testes failed to complete male differentiation and extended the period of pluripotency, they did not adopt a female fate. The expression of female marker genes such as Stra8, Foxl2, Rspo1, Ctnnb1, Bmp4, NrOb1 and Fst did not differ between control testes and Emx2-knockdown testes (Fig. S5A). We did not observe male germ cell meiosis in inhibitor-treated Emx2knockdown testes (Fig. S5B). Among the candidate signals analysed, FGF9 and NODAL signalling occurred at abnormally high levels in Emx2-knockdown testes. We hypothesize that the failure of germ cells in Emx2 knockdown testes to undergo spermatogenic differentiation is caused by persistently high levels of FGF9 secreted by Sertoli cells and its targets, the NODAL signalling genes mainly expressed in germ cells. This hypothesis is supported by rescue experiments performed on ex vivo cultured foetal gonad, in which treatment with chemical inhibitors of either FGF9 or NODAL signalling pathway down-regulated the pluripotency genes (Nanog, Sox2 and Pou5f1) and restored the expression of the differentiation marker gene Dnmt3/ in Emx2-knockdown testes. We also examined the mRNA levels of other Sertoli cell marker genes at E12.5 (Fig. S6). The mRNA levels of Cyp26b1, Ptgds, Dmrt1, Sox9, Amh, Wt1 and Gata4 in Emx2-knockdown testes were not different from those in control testes.

Our finding that EMX2 suppresses Fgf9 expression in Sertoli cells suggests a possible link between EMX2 and 
FGF signalling. However, the underlying mechanism by which EMX2 regulates Fgf9 (directly or indirectly) requires further investigation. Nanos 2 expression was up-regulated in Emx2-knockdown testes from E12.5 to E16.5 compared with the control group (Fig. S4A). This result is consistent with previous studies that FGF9 stimulates Nanos2 expression (Barrios et al. 2010, Bowles et al. 2010). Unexpectedly, the Dnmt3/ mRNA level was reduced in Emx2-knockdown testes after E13.5 (Fig. S4B), which is inconsistent with a previous report that FGF9 stimulates Dnmt3l expression (Bowles et al. 2010). We suggest that other yet-to-be-identified signals in addition to FGF9 are involved in the regulation of Dnmt3/ expression. Increased expression of Dnmt3/ is observed in Nodal knockdown gonads at E14.5 (Spiller et al. 2012). In our study, we observed that Dnmt3/ expression was significantly up-regulated after addition of the NODAL signalling inhibitor SB431542. Thus, we suggest that Nodal signalling suppresses the expression of Dnmt3l. Given the FGF9 and NODAL signalling up-regulation in Emx2-knockdown testes and their opposite roles in regulating Dnmt3/ expression, Dnmt3/ was ultimately down-regulated in the $E m \times 2$ conditional knockdown testes.

After E12.5, the expression of NODAL pathway components is reduced to allow normal male germ cell differentiation (Spiller et al. 2012). By contrast, germ cells in Emx2-knockdown testes expressed high levels of Nodal, Cripto and Lefty $1 / 2$, which restrict differentiation and contribute to pluripotency maintenance. Our conclusions regarding NODAL signalling function are complementary with the study demonstrating that Nodal knockdown leads to premature differentiation, reduced pluripotency marker expression and reduced ability to form embryonic germ (EG) cell colonies in vitro (Spiller et al. 2012). Wu et al. suggested that the $50 \%$ inhibitory concentration of SU5402 is $10-20 \mu \mathrm{M}$ (Wu et al. 2013). Our inhibitor experiments $(20 \mu \mathrm{M}$ SU5402) reduced the abnormally high level of NODAL signalling in Emx2-knockdown testes to an almost 'normal' level.

SB431542 and SU5402 inhibit TGFB1 and FGF signalling respectively (Mohammadi et al. 1997, Inman et al. 2002). The TGFB family comprises many members, including TGFB1-3, ACTIVIN and NODAL that signal through the activin-like kinases (ALK)-4, -5 and -7 to regulate target gene transcription (Young et al. 2015). The chemical inhibitor SB431542 blocks ALK-4, -5 and -7 and is not a specific inhibitor of NODAL signalling, as it also blocks TGFB1-3 and ACTIVIN signalling. In our inhibitor experiments, loss of Lefty expression confirmed that NODAL signalling was repressed; however, other TGFB1 signalling pathways may also have been inhibited. Similarly, FGF9 signalling is inhibited in company with other branches of the FGF family by the general FGFR tyrosine kinase inhibitor SU5402.
The close relationship between pluripotency and other characteristics of male germ cell differentiation has been evaluated in recent studies (Western et al. 2008, 2010, Nettersheim et al. 2011). Following the mitotic arrest of male germ cells between E12.5 and E13.5, transcriptional repression of pluripotency regulators is initiated between E13.5 and E14.5 (Western et al. 2010). Persistent expression of pluripotency regulators in germ cells is always associated with continuance of the mitotic cell cycle in the dysregulated mouse models (Krentz et al. 2009, Pesce \& Scholer 2001). Control of pluripotency in male germ cells normally involves the establishment of DNA methylation within the functional promoters of Nanog and Sox2 (Western et al. 2010). Therefore, it is particularly interesting to investigate the functional relationship between these physiological events.

In summary, this study reveals that male germ cell differentiation is arrested and FGF9/NODAL signalling occurs at abnormally high levels in Emx2-knockdown testes. Our study expands the known developmental roles of EMX2 by demonstrating its role in the pluripotency-differentiation transition and linking FGF9/NODAL pathway dysregulation with arrest of germ cell differentiation.

\section{Supplementary data}

This is linked to the online version of the paper at http://dx.doi. org/10.1530/REP-16-0022.

\section{Declaration of interest}

The authors declare that there is no conflict of interest that could be perceived as prejudicing the impartiality of the research reported.

\section{Funding}

This research was supported by the National Nature Science Foundation of China (81300484), Special Competitive Allocation Project of Science and Technology Special Financial Funding of Zhanjiang (no. 2013A1006) and The PhD Start-up Fund of Guangdong Medical College (B2012027).

\section{Author contribution statement}

YR-N conceived and designed the experiments. TZ-M, B-C and $\mathrm{Y}-\mathrm{Z}$ performed the experiments. $\mathrm{X}-\mathrm{J}, \mathrm{CL}-\mathrm{P}, \mathrm{YS}-\mathrm{Z}$ and $\mathrm{MW}-\mathrm{H}$ analysed the data. TZ-M and YR-N wrote the paper.

\section{References}

Baltus AE, Menke DB, Hu YC, Goodheart ML, Carpenter AE, de Rooij DG \& Page DC 2006 In germ cells of mouse embryonic ovaries, the decision to enter meiosis precedes premeiotic DNA replication. Nature Genetics 38 1430-1434. (doi:10.1038/ng1919) 
Barrios F, Filipponi D, Pellegrini M, Paronetto MP, Di Siena S, Geremia R, Rossi P, De Felici M, Jannini EA \& Dolci S 2010 Opposing effects of retinoic acid and FGF9 on Nanos2 expression and meiotic entry of mouse germ cells. Journal of Cell Science 123 871-880. (doi:10.1242/ jcs.057968)

Bowles J, Knight D, Smith C, Wilhelm D, Richman J, Mamiya S, Yashiro K, Chawengsaksophak K, Wilson MJ, Rossant J et al. 2006 Retinoid signaling determines germ cell fate in mice. Science 312 596-600. (doi:10.1126/science.1125691)

Bowles J, Feng CW, Spiller C, Davidson TL, Jackson A \& Koopman P 2010 FGF9 suppresses meiosis and promotes male germ cell fate in mice. Developmental Cell 19 440-449. (doi:10.1016/j.devcel.2010.08.010)

Chen SR, Chen M, Wang XN, Zhang J, Wen Q, Ji SY, Zheng QS, Gao F \& Liu YX 2013 The Wilms tumor gene, Wt1, maintains testicular cord integrity by regulating the expression of Col4a1 and Col4a2. Biology of Reproduction 88 56. (doi:10.1095/biolreprod.112.105379)

Durcova-Hills G \& Surani A 2008 Reprogramming primordial germ cells (PGC) to embryonic germ (EG) cells. Current Protocols in Stem Cell Biology Chapter 1 Unit1A 3. (doi:10.1002/9780470151808.sc01a03s5)

Hayashi S \& McMahon AP 2002 Efficient recombination in diverse tissues by a tamoxifen-inducible form of Cre: a tool for temporally regulated gene activation/inactivation in the mouse. Developmental Biology 244 305-318. (doi:10.1006/dbio.2002.0597)

Inman GJ, Nicolas FJ, Callahan JF, Harling JD, Gaster LM, Reith AD, Laping NJ \& Hill CS 2002 SB-431542 is a potent and specific inhibitor of transforming growth factor-beta superfamily type I activin receptor-like kinase (ALK) receptors ALK4, ALK5, and ALK7. Molecular Pharmacology 62 65-74. (doi:10.1124/mol.62.1.65)

Kaneda M, Okano M, Hata K, Sado T, Tsujimoto N, Li E \& Sasaki H 2004 Essential role for de novo DNA methyltransferase Dnmt3a in paternal and maternal imprinting. Nature 429 900-903. (doi:10.1038/nature02633)

Kimura T, Murayama K, Nakamura T, Watanabe S, Umehara H, Tomooka M \& Nakano T 2005 Testicular teratomas: back to pluripotent stem cells. Progress in Molecular and Subcellular Biology 40 133-150. (doi:10.1007/3-540-27671-8)

Kimura T, Tomooka M, Yamano N, Murayama K, Matoba S, Umehara $\mathrm{H}$, Kanai Y \& Nakano T 2008 AKT signaling promotes derivation of embryonic germ cells from primordial germ cells. Development $\mathbf{1 3 5}$ 869-879. (doi:10.1242/dev.013474)

Koopman P, Gubbay J, Vivian N, Goodfellow P \& Lovell-Badge R 1991 Male development of chromosomally female mice transgenic for Sry. Nature 351 117-121. (doi:10.1038/351117a0)

Koubova J, Menke DB, Zhou Q, Capel B, Griswold MD \& Page DC 2006 Retinoic acid regulates sex-specific timing of meiotic initiation in mice. PNAS 103 2474-2479. (doi:10.1073/pnas.0510813103)

Krentz AD, Murphy MW, Kim S, Cook MS, Capel B, Zhu R, Matin A, Sarver AL, Parker KL, Griswold MD et al. 2009 The DM domain protein DMRT1 is a dose-sensitive regulator of fetal germ cell proliferation and pluripotency. PNAS 106 22323-22328. (doi:10.1073/pnas.0905431106)

Kusaka M, Katoh-Fukui Y, Ogawa H, Miyabayashi K, Baba T, Shima Y, Sugiyama N, Sugimoto Y, Okuno Y, Kodama R et al. 2010 Abnormal epithelial cell polarity and ectopic epidermal growth factor receptor (EGFR) expression induced in Emx2 $\mathrm{KO}$ embryonic gonads. Endocrinology 151 5893-5904. (doi:10.1210/en.2010-0915)

Labosky PA, Barlow DP \& Hogan BL 1994 Mouse embryonic germ (EG) cell lines: transmission through the germline and differences in the methylation imprint of insulin-like growth factor 2 receptor (Igf2r) gene compared with embryonic stem (ES) cell lines. Development 120 3197-3204.

Livera G, Delbes G, Pairault C, Rouiller-Fabre V \& Habert R 2006 Organotypic culture, a powerful model for studying rat and mouse fetal testis development. Cell and Tissue Research 324 507-521. (doi:10.1007/s00441-006-0167-7)
Looijenga LH, Gillis AJ, Stoop H, Biermann K \& Oosterhuis JW 2011 Dissecting the molecular pathways of (testicular) germ cell tumour pathogenesis; from initiation to treatment-resistance. International Journal of Andrology 34 e234-e251. (doi:10.1111/j.1365-605.2011.01157.x)

Matsui Y \& Tokitake Y 2009 Primordial germ cells contain subpopulations that have greater ability to develop into pluripotential stem cells. Development Growth and Differentiation 51 657-667. (doi:10.1111/ j.1440-169X.2009.01125.x)

Matsui Y, Zsebo K \& Hogan BL 1992 Derivation of pluripotential embryonic stem cells from murine primordial germ cells in culture. Cell $\mathbf{7 0}$ 841-847. (doi:10.1016/0092-8674(92)90317-6)

Miyamoto N, Yoshida M, Kuratani S, Matsuo I \& Aizawa S 1997 Defects of urogenital development in mice lacking Emx2. Development 124 1653-1664.

Mohammadi M, McMahon G, Sun L, Tang C, Hirth P, Yeh BK, Hubbard SR \& Schlessinger J 1997 Structures of the tyrosine kinase domain of fibroblast growth factor receptor in complex with inhibitors. Science $\mathbf{2 7 6}$ 955-960. (doi:10.1126/science.276.5314.955)

Nettersheim D, Biermann K, Gillis AJ, Steger K, Looijenga LH \& Schorle H 2011 NANOG promoter methylation and expression correlation during normal and malignant human germ cell development. Epigenetics $\mathbf{6}$ 114-122. (doi:10.4161/epi.6.1.13433)

Pesce M \& Scholer HR 2001 Oct-4: gatekeeper in the beginnings of mammalian development. Stem Cells 19 271-278. (doi:10.1634/stemcells.19-4-271)

Resnick JL, Bixler LS, Cheng L \& Donovan PJ 1992 Long-term proliferation of mouse primordial germ cells in culture. Nature 359 550-551. (doi:10.1038/359550a0)

Sasaki H \& Matsui Y 2008 Epigenetic events in mammalian germ-cell development: reprogramming and beyond. Nature Reviews Genetics 9 129-140. (doi:10.1038/nrg2295)

Souquet B, Tourpin S, Messiaen S, Moison D, Habert R \& Livera G 2012 Nodal signaling regulates the entry into meiosis in fetal germ cells. Endocrinology 153 2466-2473. (doi:10.1210/en.2011-2056)

Spiller CM, Feng CW, Jackson A, Gillis AJ, Rolland AD, Looijenga LH, Koopman P \& Bowles J 2012 Endogenous Nodal signaling regulates germ cell potency during mammalian testis development. Development 139 4123-4132. (doi:10.1242/dev.083006)

Tsuda M, Sasaoka Y, Kiso M, Abe K, Haraguchi S, Kobayashi S \& Saga Y 2003 Conserved role of nanos proteins in germ cell development. Science 301 1239-1241. (doi:10.1126/science.1085222)

Western PS, Miles DC, van den Bergen JA, Burton M \& Sinclair AH 2008 Dynamic regulation of mitotic arrest in fetal male germ cells. Stem Cells 26 339-347. (doi:10.1634/stemcells.2007-0622)

Western PS, van den Bergen JA, Miles DC \& Sinclair AH 2010 Male fetal germ cell differentiation involves complex repression of the regulatory network controlling pluripotency. FASEB Journal 24 3026-3035. (doi:10.1096/fj.09-151555)

Wu Q, Kanata K, Saba R, Deng CX, Hamada H \& Saga Y 2013 Nodal/ activin signaling promotes male germ cell fate and suppresses female programming in somatic cells. Development 140 291-300. (doi:10.1242/ dev.087882)

Young JC, Wakitani S \& Loveland KL 2015 TGF-beta superfamily signaling in testis formation and early male germline development. Seminars in Cell and Developmental Biology 45 94-103. (doi:10.1016/j. semcdb.2015.10.029)

Received 14 January 2016

First decision 1 February 2016

Revised manuscript received 8 March 2016

Accepted 21 March 2016 